\title{
Approximate controllability result for nonlinear impulsive neutral fuzzy stochastic differential equations with nonlocal conditions
}

\author{
S Narayanamoorthy* and S Sowmiya
}

"Correspondence:

snm_phd@yahoo.co.in Department of Applied

Mathematics, Bharathiar University, Coimbatore, 641 046, India

\begin{abstract}
This paper deals with the approximate controllability of impulsive neutral fuzzy stochastic differential equations with nonlocal conditions in a Banach space by using the concept of fuzzy numbers whose values are normal, convex, upper semicontinuous and compact. The hypotheses are obtained by Schauder's and Banach fixed point theorems. The results are obtained by the evolution operator.
\end{abstract}

MSC: 34A07; 39A70; 93B05; 49N25

Keywords: approximate controllability; impulsive neutral fuzzy stochastic differential equations; fixed point theorems; nonlocal conditions

\section{Introduction}

In 1965, Zadeh initiated the development of the modified set theory known as fuzzy set theory, which is a tool that makes possible the description of vague notions and manipulations with them. The basic idea of fuzzy set theory is simple and natural. In [1] the authors studied the basic definitions and properties of fuzzy process, fuzzy number and so on. Stochastic differential equations are used to build more realistic models in economics, social sciences, chemistry, finance, physics and other areas. Therefore, many real world problems can be modeled by stochastic differential equations. The deterministic models often fluctuate due to noise, so we should move from deterministic control to stochastic control problems. In $[2,3]$ the basic properties and applications of stochastic differential systems and stochastic control problems were studied. The fuzzy stochastic differential equations could be applicable in the investigation of numerous engineering and economic problems where the phenomena are subjected to randomness and fuzziness simultaneously.

Neutral differential equations are one of the most thoroughly studied classes of equations with distributed arguments. They occur naturally in applied problems that contain in their statement some recurrence property and are discussed in [4-6]. Impulsive differential equations have been developed in modeling impulsive problems in physics, population dynamics, ecology, biological systems, biotechnology, industrial robotics, pharmacokinetics, optimal control, and so forth. Again associated with this development, a theory of impulsive differential equations has been given extensive attention. However, in addi-

(c) 2015 Narayanamoorthy and Sowmiya; licensee Springer. This is an Open Access article distributed under the terms of the Creative Commons Attribution License (http://creativecommons.org/licenses/by/4.0), which permits unrestricted use, distribution, and reproduction in any medium, provided the original work is properly credited. 
tion to impulsive effects, stochastic effects also exist in real systems. Therefore, impulsive stochastic differential systems describing these dynamical systems are subject to both impulsive and stochastic changes. In $[7,8]$ these types of impulsive effects and differential systems were studied.

In the literature there are only a few papers that deal with the approximate controllability of fuzzy differential systems, likewise approximate controllability of backward stochastic evolution equations in Hilbert space as discussed in [9]. Dauer et al. [10] studied the approximate controllability of semilinear functional equations in a Hilbert space. Sakthivel et $a l$. [11,12] studied the approximate controllability of nonlinear impulsive differential systems and stochastic systems with unbounded delay. Zang and Li [13] discussed the concept of approximate controllability of fraction impulsive neutral stochastic differential equations with nonlocal conditions. Kwun and Park [2] presented the optimal control problem for fuzzy differential equations and nonlocal controllability for the semilinear fuzzy integrodifferential equations in an $n$-dimensional fuzzy vector space. It should be mentioned that there is no work done on the approximate controllability of fuzzy stochastic differential systems. Motivated by the above considerations, in this paper we investigate the approximate controllability for nonlinear impulsive neutral fuzzy stochastic differential equations with nonlocal conditions described by

$$
\begin{aligned}
& d[x(t)-h(t, x(t))]=A[x(t)-h(t, x(t))]+B u(t) d t+f(t, x(t)) d t \\
& +g(t, x(t)) d w(t), \quad t \in J=[0, a], t \neq t_{i}, \\
& \Delta x\left(t_{i}\right)=x\left(t_{i}^{+}\right)-x\left(t_{i}^{-}\right) x \in X, \quad i=1,2, \ldots, m, \\
& x(0)+\mu(x)=x_{0} .
\end{aligned}
$$

Here, the state variable $x(\cdot)$ takes values in a real line separable Hilbert space $X$ with the inner product $(\cdot, \cdot)$ and the norm $\|\cdot\|$. The control function $u(\cdot)$ takes values in $L^{2}(J, U)$, a Banach space of admissible control functions for a separable Hilbert space $U$ and $J=$ $[0, b], \Omega=\{(t, s): 0 \leq s \leq t \leq b\}$. Also, $A$ (i.e., $A(t, x))$ is the infinitesimal generator of a $C_{0}$-semigroup in $X$ and $B$ is a bounded linear operator from $U$ into $X$. Further, $f$ and $g$ are continuous and compact functions and $f: J \rightarrow X$ and $g: \Omega \rightarrow X$ are measurable mappings in $X$-norm, and a neutral variable $h$ is continuous and compact; also $h: X \rightarrow X$ is a measurable mapping. And here, the function $\mu: P C(J, X) \rightarrow X$ is continuous and the impulsive function $I_{i}: X \rightarrow X$ is compact. Furthermore, the fixed time $t_{i}$ satisfies $0=t_{0}<$ $t_{1}<t_{2}<\cdots<t_{m}<a, x\left(t_{i}^{+}\right)$and $x\left(t_{i}^{+}\right)$denotes the right and left limits of $x(t)$ at $t=t_{i}$, and $\Delta x\left(t_{i}\right)=x\left(t_{i}^{+}\right)-x\left(t_{i}^{-}\right)$represents the jump in the state $X$ at time $t_{i}$, where $I_{i}$ determines the size of the jump.

\section{Preliminaries}

Here, first we define some properties, theorems and lemmas and also recall some basic definitions which are all used in this paper.

A fuzzy set of $R^{n}$ is a function $u: R^{n} \rightarrow[0,1]$. For each fuzzy set $u$, we denote by $[u]^{\alpha}=$ $x \in R^{n} ; u(x) \geq \alpha$ for any $\alpha \in[0,1]$ its $\alpha$-level set.

Let $E^{n}$ denote the collection of all fuzzy sets of $R^{n}$ that satisfies the conditions, i.e., $u$ is normal, fuzzy convex, upper semicontinuous and $[u]^{0}$ is compact. We call $u \in E^{n}$ an $n$-dimensional fuzzy number. 
And we define the abstract phase space $B_{h}$, also assume that $h:(-\infty, 0] \rightarrow(0, \infty)$ is a continuous function with $l=\int_{-\infty}^{0} h(t) d t<+\infty=\{\Psi:[-b, 0] \rightarrow X$ such that $\Psi(t)$ is bounded and measurable $\}$ and equip the space $B$ with the norm

$$
\|\Psi\|_{[-b, 0]}=\sup _{s \in[-b, 0]}|\Psi(s)| \quad \text { for all } \Psi \in B .
$$

Let us define

$$
\begin{aligned}
B_{h}= & \left\{\Psi:(-\infty, 0] \rightarrow X \text { such that for any } c>0,\left.\Psi\right|_{[-c, 0]} \in B\right. \text { and } \\
& \left.\int_{-\infty}^{0} h(s)\|\Psi\|_{[c, 0]} d s<\infty\right\} .
\end{aligned}
$$

If $B_{h}$ is endowed with the norm

$$
\|\Psi\|_{B_{h}}=\int_{-\infty}^{0} h(s)\|\Psi\|_{[s, 0]} d s \quad \text { for all } \Psi \in B_{h},
$$

then it is clear that $\left(B_{h},\|\cdot\|_{B_{h}}\right)$ is a Banach space. Now we consider the space

$$
\begin{aligned}
B_{h}^{\prime}= & \left\{x:(-\infty, b] \rightarrow X \text { such that } x_{k} \in P C\left(J_{k}, X\right) \text { and there exist } x\left(t_{k}^{+}\right)\right. \text {and } \\
& \left.x\left(t_{k}^{-}\right) \text {with } x\left(t_{k}\right)=x\left(t_{k}^{-}\right), x_{0}=\phi \in B_{h}, k=0,1, \ldots, m\right\}
\end{aligned}
$$

where $x_{k}$ is the restriction of $x$ to $J_{k}=\left(t_{k}, t_{k}+1\right], k=0,1, \ldots, m$. Set $\|\cdot\|_{h}^{\prime}$ to be a seminorm in $B_{h}^{\prime}$ defined by

$$
\|x\|_{h}^{\prime}=\|\phi\|_{B_{h}}+\sup |x(s)|: s \in[0, b], \quad x \in B_{h}^{\prime} .
$$

Definition 2.1 The family $A(t): 0 \leq t \leq b$ generates a unique linear evolution system $U(t, s): 0 \leq s \leq t \leq b$ satisfying the following properties:

- $U(t, s) U(s, \tau)=U(t, \tau)$ and $U(t, t) x=x$ for every $s \leq \tau \leq t$ and all $x \in X$,

- for every $x \in X$, the function for $(t, s)=U(t, s) x$ is continuous and $U(t, s) \in L(X)$ for every $t \geq s$, and

- for $0 \leq s \leq t \leq b$, the function $t \rightarrow U(t, s),(s, t] \in L(X)$ is differentiable with $\frac{\partial U(t, s)}{\partial t}=A(t) U(t, s)$.

Definition 2.2 Let $x, y \in C\left(I, E_{N}\right)$, here $I$ is a real interval and $E_{N}$ is the set of all upper semicontinuous convex normal fuzzy numbers with bounded $\alpha$-level intervals. A mapping $x: I \rightarrow E_{N}$ is called a fuzzy process. We define

$$
[x(t)]^{\alpha}=\left[x_{l}^{\alpha}(t), x_{r}^{\alpha}(t)\right], \quad t \in I, 0<\alpha \leq 1 .
$$

The derivative $x^{\prime}(t)$ of a fuzzy process $x$ is defined by

$$
\left[x^{\prime}(t)\right]^{\alpha}=\left[\left(x_{l}^{\alpha}\right)^{\prime}(t),\left(x_{r}^{\alpha}(t)\right)^{\prime}\right], \quad t \in I, 0<\alpha \leq 1,
$$

provided that this equation defines fuzzy $x^{\prime}(t) \in E_{N}$. 
Definition 2.3 A stochastic process $x$ is said to be a mild solution of (1)-(3) if the following conditions are satisfied:

- $X(t, \omega)$ is a measurable function from $J \times \Omega$ to $X$ and $x(t)$ is $F_{t}$-adapted,

- $E\|x(t)\|^{2}<\infty$ for all $t \in J$,

- $\Delta x\left(\tau_{i}\right)=x\left(\tau_{i}^{+}\right)-x\left(\tau_{i}^{-}\right)=I_{i}\left(x\left(\tau_{i}\right)\right), x \in X$ and $1 \leq i \leq m$,

- for each $u \in L_{2}^{F}(J, U)$, the process $x$ satisfies the following integral equation:

$$
\begin{aligned}
x(t)= & U(t, 0)\left[x^{\prime}-\mu(x)\right]+\int_{0}^{t} U(t, s) B u(s) d s+\int_{0}^{t} U(t, s) f(s, x(s)) d s \\
& +\int_{0}^{t} U(t, s) g(s, x(s)) d W(s)+\sum_{0 \leq t_{i} \leq t} U\left(t, t_{i}\right) I\left(x\left(t_{i}^{-}\right)\right), \quad t \in J .
\end{aligned}
$$

Lemma 2.4 Assume that $x \in B_{h}^{\prime}$, then for $t \in I, x_{t} \in B_{h}$. Moreover,

$$
l|x(t)| \leq\left\|x_{t}\right\|_{B_{h}} \leq\left\|x_{0}\right\|_{B_{h}}+l \sup _{s \in[0, t]}\|x(s)\|,
$$

where $l=\int_{-\infty}^{0} h(t) d t<\infty$.

Note 2.5 Define $B_{h}^{\prime \prime}=z \in B_{h}^{\prime}: z_{0}=0 \in B_{h}$. For any $z \in B_{h}^{\prime \prime}$,

$$
\|z\|_{B_{h}}^{\prime}=\left\|z_{0}\right\|_{B_{h}}+\sup \{|z(s)|: s \in[0, b]\}=\sup \{|z(s)|: s \in[0, b]\},
$$

and thus $\left(B_{h}^{\prime \prime},\|\cdot\|\right)$ is a Banach space. Set

$$
B_{r}=z \in P C((-\infty, b], X):\|z(t)\|_{B_{h}}^{\prime} \leq r, \quad 0 \leq t \leq b .
$$

Clearly, $B_{r}$ is a nonempty, bounded, convex and closed set in $B_{h}^{\prime}$. Then, for any $z \in B_{r}$, from Lemma 2.4 we have

$$
\begin{aligned}
\left\|z_{t}+y_{t}\right\|_{B_{h}} & \leq\left\|z_{t}\right\|_{B_{h}}+\left\|y_{t}\right\|_{B_{h}} \\
& \leq\left\|z_{0}\right\|_{B_{h}}+l \sup _{s \in[0, b]}|z(s)|+\left\|y_{0}\right\|_{B_{h}}+l \sup _{s \in[0, b]}|y(s)| \\
& \leq l\left(r+M_{1}|\phi(0)|\right)+\|\phi\|_{B_{h}}=r^{\prime} .
\end{aligned}
$$

For each $t \in J, z \in B_{r}$, we have by the above equation and $\left(\mathrm{A}_{2}\right)$

$$
\begin{aligned}
& \sup _{t \in J}|z(t)+y(t)| \leq l^{-1}\left\|z_{t}+y_{t}\right\|_{B_{h}} \leq l^{-1} r^{\prime}, \\
& \left|I_{k}\left(z\left(t_{k}^{-}\right)+z\left(t_{k}^{-}\right)\right)\right| \leq d_{k}\left(\sup _{t \in J}|z(t)+y(t)|\right) \leq d_{k}\left(l^{-1} r^{\prime}\right), \quad k=1,2, \ldots, m .
\end{aligned}
$$

Let $x_{b}\left(x_{0} ; u\right)$ be the state value of (1)-(3) at terminal time $b$ corresponding to the control $u$ and the initial value $x_{0}=\phi \in B_{h}$.

Introduce the set $R\left(b, x_{0}\right)=x_{b}\left(x_{0} ; u\right)(0): u(\cdot) \in L^{2}(J, U)$, which is called the reachable set at terminal time $b$, its closure in $X$ is denoted by $\overline{R\left(b, x_{0}\right)}$. 
Definition 2.6 If $\overline{R\left(b, x_{0}\right)}=X$, then system (1)-(3) is approximately controllable on the interval $J$. It is convenient at this point to define operators

$$
\begin{aligned}
& \Gamma_{0}^{a}=\int_{0}^{a} U(a, s) B B^{*} U^{*}(a, s) d s, \\
& R\left(\alpha, \Gamma_{0}^{a}\right)=\left(\alpha I+\Gamma_{0}^{a}\right)^{-1} . \\
& \text { (D) } \alpha R\left(\alpha, \Gamma_{0}^{a}\right) \rightarrow 0 \text { as } \alpha \rightarrow 0^{+} \text {is strong operator topology. }
\end{aligned}
$$

It is known that assumption (D) holds iff the linear system $\dot{x}(t)=A x(t)+B u(t), x(0)=x_{0}$ is approximately controllable on $J$.

The following theorem gives a formula for a control transferring the initial state $x_{0}$ to some neighborhood of $x_{a}$ at time $a$.

Theorem 2.7 For arbitrary $x_{a} \in X$, the control

$$
u(t)=B^{*} U^{*}(a, t) R\left(\alpha, \Gamma_{0}^{a}\right) p(x(\cdot))
$$

where

$$
\begin{aligned}
p(x(\cdot))= & x_{a}-U(a, 0)\left[x_{0}-\mu(x)-h\left(0,\left(x_{0}-\mu(x)\right)\right)\right]-h\left(a, x_{a}\right) \\
& -\int_{0}^{a} U(a, s) A(s) h\left(s, x_{s}\right) d s-\int_{0}^{a} U(a, s) f\left(s, x_{s}\right) d s \\
& -\int_{0}^{a} U(a, s) g\left(s, x_{s}\right) d W(s)-\sum_{k=1}^{m} U\left(a, t_{k}\right)\left(I_{k}\right)\left(x\left(t_{k}^{-}\right)\right)
\end{aligned}
$$

transfers the initial state $x_{0}$ to

$$
\begin{aligned}
x(a)= & x_{a}-\alpha\left(\alpha I+\Gamma_{0}^{a}\right)^{-1}\left[x_{a}-U(a, 0)\left[x_{0}-\mu(x)-h\left(0,\left(x_{0}-\mu(x)\right)\right)\right]-h\left(a, x_{a}\right)\right. \\
& -\int_{0}^{a} U(a, s) A(s) h\left(s, x_{s}\right) d s-\int_{0}^{a} U(a, s) f\left(s, x_{s}\right) d s-\int_{0}^{a} U(a, s) g\left(s, x_{s}\right) d W(S) \\
& \left.-\sum_{k=1}^{m} U\left(a, t_{k}\right)\left(I_{k}\right)\left(x\left(t_{k}^{-}\right)\right)\right] .
\end{aligned}
$$

\section{Approximate controllability}

In this section, we state the approximate controllability result for impulsive neutral fuzzy stochastic differential equations under nonlocal conditions by using Schauder's fixed point theorem. Approximate controllability property is the possibility to steer the state of the system from any initial data to a state of arbitrary close to a target by choosing a suitable control.

To establish the result, we introduce the following assumptions on system (1)-(3).

$\left(\mathrm{A}_{1}\right)$ When $t>s>0$, then the strongly continuous semigroup of bounded linear operators $U(t, s)$ generated by $A(t)$ is compact and there exist constants $M_{1}>0, M_{2}>0$ such 
that

$$
\left\|[U(t, s)]^{\alpha}\right\| \leq M_{1}^{\alpha}, \quad\left\|[U(t, s)]^{\alpha}\right\| \leq M_{2}^{\alpha}
$$

$\left(\mathrm{A}_{2}\right)$ (i) The nonlinear function $f: J \times B_{h} \times X \rightarrow X$ is continuous, uniformly bounded, strongly measurable and there exists $L_{f}>0$ such that

$$
\left\|[f(t, \phi, x)]^{\alpha}\right\| \leq L_{f}^{\alpha} \quad \text { for all }(t, \phi, x) \in J \times B_{h} \times X .
$$

(ii) For each positive number $r$, there exists a function $\delta_{r} \in L^{1}\left(J, R_{+}\right)$such that

$$
\begin{aligned}
& \sup \left\{[f(t, \phi, x)]^{\alpha}:\|\phi\|_{B_{h}} \leq r,\|[x]\| \leq r\right\} \leq \delta_{r}(t) \quad \text { for a.e. } t \in J \text { and } \\
& \liminf _{r \rightarrow \infty} \frac{1}{r} \int_{0}^{a} \delta_{r}(s) d s=\gamma<\infty .
\end{aligned}
$$

$\left(\mathrm{A}_{3}\right)$ The nonlinear function $g: J \times B_{h} \times X \rightarrow X$ is continuous, strongly measurable and there exists $L_{g}>0$ such that

$$
\left\|[g(t, \phi, x)]^{\alpha}\right\| \leq L_{g}^{\alpha} \quad \text { for all }(t, \phi, x) \in J \times B_{h} \times X .
$$

$\left(\mathrm{A}_{4}\right)$ The function $\mu: P C(J, X) \rightarrow X$ is a nonlocal condition in the following sense, and there exists a constant $L_{\mu}>0$ such that

$$
\left\|[\mu(t, u)]^{\alpha}-[\mu(s, v)]^{\alpha}\right\| \leq L_{\mu}^{\alpha}[|t-s|-\|u-v\|] \quad \text { for all } u, v \in X .
$$

( $\left.\mathrm{A}_{5}\right) I_{k} \in C(X, X)$ and there exists a constant $d_{k}^{\alpha}$ such that

$$
\left|\left[I_{k}(x)\right]^{\alpha}\right| \leq d_{k}^{\alpha}(|x|), \quad k=1,2, \ldots, m,
$$

for each $x \in X$, and

$$
\liminf \frac{d_{k}(\rho)}{\rho}=\lambda_{k}<\infty, \quad k=1,2, \ldots, m
$$

(A6) The function $h: J \times B_{h} \rightarrow X$ is continuous, and there exists $L_{h}>0$ such that

$$
\left\|\left[h\left(t, \phi_{1}\right)\right]^{\alpha}-\left[h\left(s, \phi_{2}\right)\right]^{\alpha}\right\|<L_{h}^{\alpha}\left[|t-s|+\left\|\phi_{1}-\phi_{2}\right\|_{B_{h}}\right] \quad \text { for every } t, s \in J \text { and } \phi_{1}, \phi_{2} \in B_{h} .
$$

The following notations are introduced for convenience:

$$
Q^{\prime}=\frac{L_{1}^{\alpha} q^{\prime}}{M_{1}^{\alpha}}+a M_{1}^{\alpha} N_{1}^{\alpha} q^{\prime}+M_{1}^{\alpha} \int_{0}^{a} \lambda_{q}^{\alpha}(s) d s+M_{1}^{\alpha} \int_{0}^{a} \lambda_{q}^{\alpha}(s) d W(s)+M_{1}^{\alpha} \sum_{k=1}^{m} d_{k}^{\alpha}\left(q^{\prime}\right)
$$

Theorem 3.1 Assume that conditions $\left(\mathrm{A}_{1}\right)-\left(\mathrm{A}_{6}\right)$ are satisfied. Further, suppose that for all $a>0$,

$$
\left(1+\frac{1}{\beta} a\left[M_{1}^{2}\right]^{\alpha}\left[M_{A}^{2}\right]^{\alpha}\right) M_{1} Q^{\prime}<1,
$$

then system (1)-(3) has a solution on J. 
Proof Now, define the operator $\Re$ on $P C((-\infty, b], X),(\Re x)(t)=\phi(t), t \in(-\infty, 0]$.

From the theorem and definition, let

$$
\begin{aligned}
{[u(t)]^{\alpha}=} & {\left[u_{l}^{\alpha}(t), u_{r}^{\alpha}(t)\right] } \\
= & {\left[B ^ { * } U ^ { * } ( a , t ) ( \alpha I + \Gamma _ { 0 } ^ { a } ) ^ { - 1 } \left(x_{a}-U_{l}^{\alpha}(a, 0)\left[x_{0}-\mu(x)-h_{l}^{\alpha}\left(0,\left(x_{0}-\mu(x)\right)\right)\right]\right.\right.} \\
& -h_{l}^{\alpha}(a, x(a))-\int_{0}^{a} U_{l}^{\alpha}(a, s) A(s) h_{l}^{\alpha}(s, x(s)) d s-\int_{0}^{a} U_{l}^{\alpha}(a, s) f_{l}^{\alpha}(s, x(s)) d s \\
& \left.-\int_{0}^{a} U_{l}^{\alpha}(a, s) g_{l}^{\alpha}(s, x(s)) d W(S)-\sum_{k=1}^{m} U_{l}^{\alpha}\left(I_{k}\right)_{l}^{\alpha}\left(x\left(t_{k}^{-}\right)\right)\right), B^{*} U^{*}(a, t) \\
& \times\left(\alpha I+\Gamma_{0}^{a}\right)^{-1}\left(x_{a}-U_{r}^{\alpha}(a, 0)\left[x_{0}-\mu(x)-h_{r}^{\alpha}\left(0,\left(x_{0}-\mu(x)\right)\right)\right]-h_{r}^{\alpha}(a, x(a))\right. \\
& -\int_{0}^{a} U_{r}^{\alpha}(a, s) A(s) h_{r}^{\alpha}(s, x(s)) d s-\int_{0}^{a} U_{r}^{\alpha}(a, s) f_{r}^{\alpha}(s, x(s)) d s \\
& \left.\left.-\int_{0}^{a} U_{r}^{\alpha}(a, s) g_{r}^{\alpha}(s, x(s)) d W(S)-\sum_{k=1}^{m} U_{r}^{\alpha}\left(I_{k}\right)_{r}^{\alpha}\left(x\left(t_{k}^{-}\right)\right)\right)\right]
\end{aligned}
$$

Take the function $y(\cdot):(-\infty, a) \rightarrow X$ defined by

$$
y(t)=\{U(t, 0) X(0) t \geq 0, X(0)-\infty<t<0\},
$$

then $y_{0}=\phi$, and the map $t \rightarrow y_{t}$ is continuous. If $[x(t)]^{\alpha}$ is a fuzzy solution of (4), then we can write it as $[x(t)]^{\alpha}=[y(t)]^{\alpha}+[z(t)]^{\alpha},-\infty<t \leq a$.

From the above we describe $Z_{0}=0$ and the function $[z(t)]^{\alpha}$ that satisfies

$$
\begin{aligned}
{[z(t)]^{\alpha}=} & {\left[-U(t, 0) h\left(0,\left[x_{0}-\mu(x)\right]\right)+h\left(t, z_{t}+y_{t}\right)+\int_{0}^{t} U(t, s) A(s) h\left(s, z_{s}+y_{s}\right) d s\right.} \\
& +\int_{0}^{t} U(t, \eta) B B^{*} U^{*}(a, s)\left(\alpha I+\Gamma_{0}^{a}\right)^{-1} \\
& \times\left(x_{a}-U(a, 0)\left[x_{0}-\mu(x)-h\left(0,\left(x_{0}-\mu(x)\right)\right)\right]\right. \\
& -h\left(a, z_{a}+y_{a}\right)-\int_{0}^{a} U(a, s) A(s) h\left(s, z_{s}+y_{s}\right) d s-\int_{0}^{a} U(a, s) f\left(s, Z_{s}+y_{s}\right) d s \\
& \left.-\int_{0}^{a} U(a, s) g\left(s, z_{s}+y_{s}\right) d W(s)-\sum_{k=1}^{m} U\left(I_{k}\right)\left(z\left(t_{k}^{-}\right)+y\left(t_{k}^{-}\right)\right)\right) \eta d \eta \\
& +\int_{0}^{t} U(t, s) f\left(s, z_{s}+y_{s}\right) d s+\int_{0}^{t} U(t, s) g\left(s, z_{s}+y_{s}\right) d W(s) \\
& \left.+\sum_{0<t_{k}<t} U\left(t, t_{k}\right) I_{k}\left(z\left(t_{k}^{-}\right)+y\left(t_{k}^{-}\right)\right)\right]^{\alpha}
\end{aligned}
$$


iff $x$ satisfies

$$
\begin{aligned}
{[x(t)]^{\alpha}=} & {\left[-U(t, 0) h\left(0,\left[x_{0}-\mu(x)\right]\right)+h\left(t, x_{t}\right)+\int_{0}^{t} U(t, s) A(s) h\left(s, x_{s}\right) d s\right.} \\
& +\int_{0}^{t} U(t, \eta) B B^{*} U^{*}(a, s)\left(\alpha I+\Gamma_{0}^{a}\right)^{-1} \\
& \times\left(x_{a}-U(a, 0)\left[x_{0}-\mu(x)-h\left(0, x_{0}-\mu(x)\right)\right]\right. \\
& -h\left(a, x_{a}\right)-\int_{0}^{a} U(a, s) A(s) h\left(s, x_{s}\right) d s-\int_{0}^{a} U(a, s) f\left(s, x_{s}\right) d s \\
& \left.-\int_{0}^{a} U(a, s) g\left(s, x_{s}\right) d W(s)-\sum_{k=1}^{m} U\left(I_{k}\right)\left(x\left(t_{k}^{-}\right)\right)\right) \eta d \eta \\
& \left.+\int_{0}^{t} U(t, s) f\left(s, x_{s}\right) d s+\int_{0}^{t} U(t, s) g\left(s, x_{s}\right) d W(s)+\sum_{0<t_{k}<t} U\left(t, t_{k}\right) I_{k}\left(x\left(t_{k}^{-}\right)\right)\right]^{\alpha}
\end{aligned}
$$

and

$$
[x(t)]^{\alpha}=\phi^{\alpha}(t), \quad \text { for all } t \in(-\infty, 0] .
$$

Let us take the operator $\Psi: B_{h}^{\prime \prime} \rightarrow B_{h}^{\prime \prime}$ defined by

$$
\begin{aligned}
& {[(\Psi z)(t)]^{\alpha}=0, \quad t \in(-\infty, 0],} \\
& {[(\Psi z)(t)]^{\alpha}=[z(t)]^{\alpha}, \quad t \in J .}
\end{aligned}
$$

Obviously, the operator $\Re$ has a fixed point iff $\Psi$ has a fixed point. So we want to prove that $\Psi$ has a fixed point and the proof has the following steps.

Step 1: $\Psi\left(B_{q}\right) \subseteq B_{q}$ for some $q>0$.

We claim that there exists a positive constant $q$ such that

$$
\Psi\left(B_{q}\right) \subseteq B_{q}
$$

If this condition is false, then for every positive number $q$, there exists a function $z^{q}(\cdot) \in$ $B_{q}$, but $\Psi\left(z^{q}\right)$ does not belong to $B_{q}$, i.e., $\left|\left(\psi z^{q}\right)(t)\right|>q$ for some $t \in J$. From conditions $\left(\mathrm{A}_{1}\right)-\left(\mathrm{A}_{6}\right)$ and

$$
\begin{aligned}
q< & \left|\left[\left(\Psi z^{q}\right)(t)\right]^{\alpha}\right| \\
\leq & M_{1}^{\alpha}\left\|h^{\alpha}\left(0, x_{0}-\mu(x)\right)\right\|+\left\|h^{\alpha}\left(t, z_{t}^{q}+y_{t}^{q}\right)\right\|+M_{1}^{\alpha} \int_{0}^{t}\left\|A(s) h^{\alpha}\left(s, z_{s}^{q}+y_{s}^{q}\right)\right\| \\
& +\int_{0}^{t} \frac{1}{\beta}\left[M_{1}^{2}\right]^{\alpha}\left[M_{A}^{2}\right]^{\alpha}\left[\left\|x_{a}\right\|+M_{1}^{\alpha}\left\|\left[x_{0}-\mu(x)\right]^{\alpha}\right\|+M_{1}^{\alpha}\left\|h^{\alpha}\left(0,\left[x_{0}-\mu(x)\right]\right)\right\|\right. \\
& +\left\|h^{\alpha}\left(a, z_{a}^{q}+y_{a}^{q}\right)\right\|+M_{1}^{\alpha} \int_{0}^{a}\left\|A(s) h^{\alpha}\left(s, z_{s}^{q}+y_{s}^{q}\right)\right\| d s+M_{1}^{\alpha} \int_{0}^{a}\left\|f^{\alpha}\left(s, z_{s}^{q}+y_{s}^{q}\right)\right\| d s
\end{aligned}
$$




$$
\begin{aligned}
& \left.+M_{1}^{\alpha} \int_{0}^{a}\left\|g^{\alpha}\left(s, z_{s}^{q}+y_{s}^{q}\right)\right\| d W(s)+M_{1}^{\alpha} \sum_{k=1}^{m}\left\|I^{\alpha}-k\left(z^{q}\left(t_{k}^{-}\right)+y^{q}\left(t_{k}^{-}\right)\right)\right\|\right] \\
& +M_{1}^{\alpha} \int_{0}^{t}\left\|f^{\alpha}\left(s, z_{s}^{q}+y_{s}^{q}\right)\right\| d s+M_{1}^{\alpha} \int_{0}^{t}\left\|g^{\alpha}\left(s, z_{s}^{q}+y_{s}^{q}\right)\right\| d W(s) \\
& +M_{1}^{\alpha} \sum_{0<t_{k}<t}\left\|I_{k}^{\alpha}\left(z^{q}\left(t_{k}^{-}\right)+y^{q}\left(t_{k}^{-}\right)\right)\right\| .
\end{aligned}
$$

Since

$$
\int_{0}^{t}\left\|f^{\alpha}\left(s, z_{s}^{q}+y_{s}^{q}\right)\right\| d s \leq \int_{0}^{a} \lambda_{q}^{\alpha}(t) d t
$$

similarly

$$
\int_{0}^{t}\left\|g^{\alpha}\left(s, z_{s}^{q}+y_{s}^{q}\right)\right\| d W(s) \leq \int_{0}^{a} \lambda_{q}^{\alpha}(t) d W(t)
$$

From conditions $\left(A_{2}\right),\left(A_{3}\right)$ and Note 2.5 , then

$$
\begin{aligned}
q< & M_{1}^{\alpha}\left\|h^{\alpha}\left(0, x_{0}-\mu(x)\right)\right\|+L_{1}^{\alpha} q^{\prime}+L_{2}^{\alpha}+a M_{1}^{\alpha} N_{1}^{\alpha} q^{\prime}+N_{2}^{\alpha} \\
& +\frac{1}{\beta}\left[M_{1}^{2}\right]^{\alpha}\left[M_{A}^{2}\right]^{\alpha}\left[\left\|x_{a}\right\|+M_{1}^{\alpha}\left\|\left[x_{0}-\mu(x)\right]^{\alpha}\right\|+M_{1}^{\alpha}\left\|h^{\alpha}\left(0,\left[x_{0}-\mu(x)\right]\right)\right\|\right. \\
& +L_{1}^{\alpha} q^{\prime}+L_{2}^{\alpha}+a M_{1}^{\alpha} N_{1}^{\alpha} q^{\prime}+N_{2}^{\alpha}+M_{1}^{\alpha} \int_{0}^{a} \lambda_{q}^{\alpha}(s) d s+M_{1}^{\alpha} \int_{0}^{a} \lambda_{q}^{\alpha}(s) d W(s) \\
& \left.+M_{1}^{\alpha} \sum_{k=1}^{m} d_{k}^{\alpha}\left(q^{\prime}\right)\right]+M_{1}^{\alpha} \int_{0}^{a} \lambda_{q}^{\alpha}(s) d s+M_{1}^{\alpha} \int_{0}^{a} \lambda_{q}^{\alpha}(s) d W(s)+M_{1}^{\alpha} \sum_{k=1}^{m} d_{k}^{\alpha}\left(q^{\prime}\right) \\
\leq & +\left(1+\frac{1}{\beta}\left[M_{1}^{2}\right]^{\alpha}\left[M_{A}^{2}\right]^{\alpha}\right)\left[L_{1}^{\alpha} q^{\prime}+a M_{1}^{\alpha} N_{1}^{\alpha} q^{\prime}+M_{1}^{\alpha} \int_{0}^{a} \lambda_{q}^{\alpha}(s) d s\right. \\
& \left.+M_{1}^{\alpha} \int_{0}^{a} \lambda_{q}^{\alpha}(s) d W(s)+M_{1}^{\alpha} \sum_{k=1}^{m} d^{\alpha}-k\left(q^{\prime}\right)\right],
\end{aligned}
$$

where $Q$ is independent of $q$. Dividing both sides by $q$, we obtain

$$
\begin{aligned}
& \lim _{q \rightarrow+\infty} \inf \sum_{k=1}^{m} \frac{d^{\alpha}-k\left(l^{-1} q^{\prime}\right)}{q}=\lim _{q \rightarrow+\infty} \inf \sum_{k=1}^{m} \frac{d_{k}^{\alpha}\left(l^{-1} q^{\prime}\right)}{\left(l^{-1} q^{\prime}\right)} \frac{l^{-1} q^{\prime}}{q}, \\
& \lim _{q \rightarrow+\infty} \inf \frac{1}{q} \int_{0}^{a} \lambda_{q}^{\alpha}(s) d s=\lim _{q \rightarrow+\infty} \inf \frac{\int_{0}^{a} \lambda_{q}^{\alpha}(s) d s}{q}=\left(1+L^{0}\right) \Gamma l .
\end{aligned}
$$

Thus we have

$$
\left(1+\frac{1}{\beta} a\left[M_{1}^{2}\right]^{\alpha}\left[M_{A}^{2}\right]^{\alpha}\right) M_{1} Q^{\prime} \geq 1
$$

This contradicts (9). Hence for $q>0, \Psi\left(B_{q}\right) \subseteq B_{q}$.

Step 2: $\Psi$ maps $B_{q}$ into a precompact set in X. 
Now we want to prove that $V(t)=(\Psi z)(t): z \in B_{q}$ is relatively compact in $X$. The case $t=0$ is true since $V(0)=\Psi(0)$. Let $0<t \leq a$ be fixed, $0<\epsilon<t$, for $z \in B_{q}$, we define

$$
\begin{aligned}
{\left[\left(\Psi_{\epsilon} z\right)(t)\right]^{\alpha}=} & {\left[-U(t, 0) h\left(0,\left[x_{0}-\mu(x)\right]\right)+h\left(t, z_{t}+y_{t}\right)\right.} \\
& +U(t, t-\epsilon) \int_{0}^{t-\epsilon} U(t-\epsilon, s) A(s) h\left(s, z_{s}+y_{s}\right) d s \\
& +U(t, t-\epsilon) \int_{0}^{t-\epsilon} U(t-\epsilon, \eta) B B^{*} U^{*}(t-\epsilon, \eta)\left(\alpha I+\Gamma_{0}^{a}\right)^{-1} \\
& \times\left(x_{a}-U(a, 0)\left[x_{0}-\mu(x)-h\left(0, x_{0}-\mu(x)\right)\right]-h\left(a, z_{a}+y_{a}\right)\right. \\
& -\int_{0}^{a} U(a, s) A(s) h\left(s, z_{s}+y_{s}\right) d s-\int_{0}^{a} U(a, s) f\left(s, z_{s}+y_{s}\right) d s \\
& \left.-\int_{0}^{a} U(a, s) g\left(s, z_{s}+y_{s}\right) d W(s)-\sum_{k=1}^{m} U\left(I_{k}\right)\left(z\left(t_{k}^{-}\right)+y\left(t_{k}^{-}\right)\right)\right) \eta d \eta \\
& +U(t, t-\epsilon) \int_{0}^{t-\epsilon} U(t-\epsilon, s) f\left(s, z_{s}+y_{s}\right) d s \\
& +U(t, t-\epsilon) \int_{0}^{t-\epsilon} U(t-\epsilon, s) g\left(s, z_{s}+y_{s}\right) d W(s) \\
& \left.+\sum_{0<t_{k}<t} U\left(t, t_{k}\right) I_{k}\left(z\left(t_{k}^{-}\right)+y\left(t_{k}^{-}\right)\right)\right]^{\alpha} .
\end{aligned}
$$

Set $V_{\epsilon}(t)=\left(\Psi_{\epsilon} z\right)(t): z \in B_{q}$ is relatively compact in $X$ for every $\epsilon, 0<\epsilon<t$. That is, a finite set $\tilde{y}_{j}, 1 \leq j \leq m$ in $X$ exists such that

$$
V_{\epsilon}(t) \subset \bigcup_{j=0}^{m} \tilde{L}\left(\tilde{y}_{j}, \eta / 2\right),
$$

where $\tilde{L}\left(\tilde{y}_{j}, \eta / 2\right)$ is an open ball in $X$ with radius $\eta / 2$ and center at $\tilde{y}_{j}$.

$$
\begin{aligned}
& \left\|[(\Psi z)(t)]^{\alpha}-\left[\left(\Psi_{\epsilon} z\right)(t)\right]^{\alpha}\right\| \\
& \leq \int_{t-\epsilon}^{t}\left\|U(t, s) A(s) h\left(s, z_{s}+y_{s}\right)\right\| d s \\
& \quad+\int_{t-\epsilon}^{t} U(t, \eta) B B^{*} U^{*}(t, \eta)\left(\alpha I+\Gamma_{0}^{a}\right)^{-1}\left[x_{a}-U(a, 0)\left[\left(x_{0}-\mu(x)\right)\right.\right. \\
& \left.\quad-h\left(0, x_{0}-\mu(x)\right)\right]-h\left(a, z_{a}+y_{a}\right)-\int_{0}^{a} U(a, s) A(s) h\left(s, z_{s}+y_{s}\right) d s \\
& \quad-\int_{0}^{a} U(a, s) f\left(s, Z_{s}+y_{s}\right) d s-\int_{0}^{a} U(a, s) g\left(s, z_{s}+y_{s}\right) d W(s) \\
& \left.\quad-\sum_{k=1}^{m} U\left(a, t_{k}\right) I_{k}\left(z\left(t_{k}^{-}\right)+y\left(t_{k}^{-}\right)\right)\right] \eta d \eta+\int_{t-\epsilon}^{t}\left\|U(t, s) f\left(s, z_{s}+y_{s}\right)\right\| d s
\end{aligned}
$$




$$
\begin{aligned}
& +\int_{t-\epsilon}^{t}\left\|U(t, s) g\left(s, z_{s}+y_{s}\right)\right\| d W(s) \\
\leq & M_{1}^{\alpha} \int_{t-\epsilon}^{t}\left[N_{1}^{\alpha} q^{\prime}+N_{2}^{\alpha}\right] d s+\frac{1}{\beta}\left[M_{1}^{2}\right]^{\alpha}\left[M_{A}^{2}\right]^{\alpha} \\
& \times \int_{t-\epsilon}^{t}\left[\left\|x_{a}\right\|+M_{1}^{\alpha}\left\|x_{0}-\mu(x)\right\|+M_{1}^{\alpha}\left\|h^{\alpha}\left(0, x_{0}-\mu(x)\right)\right\|\right. \\
& +L_{1}^{\alpha} q^{\prime}+L_{2}^{\alpha}+a M_{1}^{\alpha}\left[N_{1}^{\alpha} q^{\prime}+N_{2}^{\alpha}\right]+M_{1}^{\alpha} \int_{0}^{a} \lambda_{q}^{\alpha}(\tau) d \tau+M_{1}^{\alpha} \int_{0}^{a} \lambda_{q}^{\alpha}(\tau) d W(\tau) \\
& \left.+M_{1}^{\alpha} \sum_{k=1}^{m} d_{k}^{\alpha}\left(l^{-1} q^{\prime}\right)\right] d s+M_{1}^{\alpha} \int_{0}^{a} \lambda_{q}^{\alpha}(s) d s+M_{1}^{\alpha} \int_{0}^{a} \lambda_{q}^{\alpha}(s) d W(s) \\
\leq & \eta / 2 .
\end{aligned}
$$

Similarly,

$$
V(t) \subset \bigcup_{j=0}^{m} \tilde{L}\left(\tilde{y}_{j}, \eta\right)
$$

Hence, for every $t \in[0, a]$, the set $V(t)$ is relatively compact in $X$.

Step 3: $\Psi$ maps $B_{q}$ into an equicontinuous family.

We claim that $\Psi\left(B_{q}\right)=\Psi z: z \in B_{q}$ is a family of equicontinuous functions. Let $t_{1}, t_{2} \in$ $[0, a]$. By $\left(\mathrm{A}_{2}\right)-\left(\mathrm{A}_{6}\right)$, we get

$$
\begin{aligned}
& \left|\left[(\Psi z)\left(t_{1}\right)\right]^{\alpha}-\left[(\Psi z)\left(t_{2}\right)\right]^{\alpha}\right| \\
& \leq\left\|\left[U^{\alpha}\left(t_{1}, 0\right)-U^{\alpha}\left(t_{2}, 0\right)\right] h^{\alpha}\left(0,\left[x_{0}-\mu(x)\right]\right)\right\|+\| h^{\alpha}\left(t_{1}, z_{t_{1}}+y_{t_{1}}\right) \\
& \quad-h^{\alpha}\left(t_{2}, z_{t_{2}}+y_{t_{2}}\right)\|+\| \int_{0}^{t_{1}}\left[U^{\alpha}\left(t_{1}, s\right)-U^{\alpha}\left(t_{2}, s\right)\right] A(s) h^{\alpha}\left(s, z_{s}+y_{s}\right) d s \| \\
& \quad+\left\|\int_{t_{1}}^{t_{2}} U^{\alpha}\left(t_{2}, s\right) A(s) h^{\alpha}\left(s, z_{s}+y_{s}\right) d s\right\|+\left\|\int_{0}^{t_{1}}\left[U^{\alpha}\left(t_{1}, \eta\right)-U^{\alpha}\left(t_{2}, \eta\right)\right] B U(s) d s\right\| \\
& \quad+\left\|\int_{t_{1}}^{t_{2}} U^{\alpha}\left(t_{2}, \eta\right) B U(s) d s\right\|+\left\|\int_{0}^{t_{1}}\left[U^{\alpha}\left(t_{1}, s\right)-U^{\alpha}\left(t_{2}, s\right)\right] f^{\alpha}\left(s, Z_{s}+y_{s}\right) d s\right\| \\
& \quad+\left\|\int_{t_{1}}^{t_{2}} U^{\alpha}\left(t_{2}, s\right) f^{\alpha}\left(s, Z_{s}+y_{s}\right) d s\right\|+\left\|\int_{0}^{t_{1}}\left[U^{\alpha}\left(t_{1}, s\right)-U^{\alpha}\left(t_{2}, s\right)\right] g^{\alpha}\left(s, Z_{s}+y_{s}\right) d s\right\| \\
& \quad+\left\|\int_{t_{1}}^{t_{2}} U^{\alpha}\left(t_{2}, s\right) g^{\alpha}\left(s, Z_{s}+y_{s}\right) d W(s)\right\| \\
& \quad+\left\|\sum_{0<t_{k}<t}\left[U^{\alpha}\left(t_{2}, t_{k}\right)-U^{\alpha}\left(t_{1}, t_{k}\right)\right] I_{k}\left(z\left(t_{k}^{-}\right)+y\left(t_{k}^{-}\right)\right)\right\| \\
& \quad+\left\|\sum_{t_{1}<t_{k}<t_{2}} U^{\alpha}\left(t_{2}, t_{k}\right) I_{k}\left(z\left(t_{k}^{-}\right)+y\left(t_{k}^{-}\right)\right)\right\| \\
& \leq
\end{aligned}
$$




$$
\begin{aligned}
& +\int_{t_{1}}^{t_{2}}\left\|U^{\alpha}\left(t_{2}, s\right)\right\|\left[N_{1}^{\alpha}\left\|z_{s}+y_{s}\right\|_{B_{h}}+N_{2}^{\alpha}\right] d s \\
& +\frac{1}{\beta}\left[M_{1}^{2}\right]^{\alpha}\left[M_{A}^{2}\right]^{\alpha} \int_{0}^{t_{1}}\left\|U^{\alpha}\left(t_{1}, \eta\right)-U^{\alpha}\left(t_{2}, \eta\right)\right\|\left[\left\|x_{a}\right\|+M_{1}^{\alpha}\left|\left[x_{0}-\mu(x)\right]\right|\right. \\
& +\left|h^{\alpha}\left(0,\left[x_{0}-\mu(x)\right]\right)\right|+\left[L_{1}^{\alpha}\left\|z_{a}+y_{a}\right\|_{B_{h}}+L_{2}^{\alpha}\right]+M_{1}^{\alpha} \int_{0}^{a}\left[N_{1}^{\alpha}\left\|z_{s}+y_{s}\right\|_{B_{h}}+N_{2}^{\alpha}\right] d s \\
& \left.+M_{1}^{\alpha}\left(\int_{0}^{a} \lambda_{q}^{\alpha}(s) d s+\int_{0}^{a} \lambda_{q}^{\alpha}(s) d W(s)+\sum_{k=1}^{m} d_{k}^{\alpha}\left(l^{-1} q^{\prime}\right)\right)\right] d \eta \\
& +\frac{1}{\beta}\left[M_{1}^{2}\right]^{\alpha}\left[M_{A}^{2}\right]^{\alpha} \int_{t_{1}}^{t_{2}}\left\|U^{\alpha}\left(t_{2}, \eta\right)\right\| \\
& +\left[\left\|x_{a}\right\|+M_{1}^{\alpha}\left|\left[x_{0}-\mu(x)\right]\right|+\left|h^{\alpha}\left(0,\left[x_{0}-\mu(x)\right]\right)\right|+\left[L_{1}^{\alpha}\left\|z_{a}+y_{a}\right\|_{B_{h}}+L_{2}^{\alpha}\right]\right. \\
& +\int_{0}^{t_{1}}\left\|U^{\alpha}\left(t_{1}, s\right)-U^{\alpha}\left(t_{2}, s\right)\right\| \lambda_{q}^{\alpha}(s) d W(s)+\int_{t_{1}}^{t_{2}}\left\|U^{\alpha}\left(t_{2}, s\right)\right\| \lambda_{q}^{\alpha}(s) d W(s) \\
& +\sum_{0<t_{k}<t}\left\|U^{\alpha}\left(t_{1}, t_{k}\right)-U^{\alpha}\left(t_{2}, t_{k}\right)\right\| d_{k}^{\alpha}\left(l^{-1} q^{\prime}\right)+M_{1}^{\alpha} \sum_{t_{1}<t_{k}<t_{2}} d_{k}^{\alpha}\left(l^{-1} q^{\prime}\right) . \\
& +\int_{t_{1}}^{t_{2}}\left\|U^{\alpha}\left(t_{2}, s\right)\right\| \lambda_{q}^{\alpha}(s) d s \\
& \left.\left.+M_{1}^{\alpha} \sum_{k=1}^{m} d_{k}^{\alpha}\left(l^{-1} q^{\prime}\right)\right] d \eta+z_{s}+y_{s} \|_{B_{h}}+N_{2}^{\alpha}\right] d s+M_{1}^{\alpha}\left(t_{1}, s\right)-U_{0}^{\alpha}\left(t_{2}, s\right) \| \lambda_{q}^{\alpha}(s) d s \\
& +\lambda_{q}^{\alpha}(s) d W(s)
\end{aligned}
$$

This implies that $\left|\left[(\Psi z)\left(t_{1}\right)\right]^{\alpha}-\left[(\Psi z)\left(t_{2}\right)\right]^{\alpha}\right| \rightarrow 0$ as $t_{2}-t_{1} \rightarrow 0$, since the compactness of $U^{\alpha}(t, s)$ is the continuity of $U^{\alpha}(t, s)$ in the uniform operator topology. Thus $\Psi$ maps $B_{q}$ into an equicontinuous family of functions.

Step 4: $B_{h}^{\prime \prime} \rightarrow B_{h}^{\prime \prime}$ is continuous.

First we show that $\Psi$ is continuous in $B_{h}^{\prime \prime}$. Let $\left[z_{t}^{(n)}(t)\right]^{\alpha}{ }_{0}^{\infty} \subseteq B_{h}^{\prime \prime}$ with $z_{n} \rightarrow z$ in $B_{h}^{\prime \prime}$. Then there exists a number $q>0$ such that $\left|\left[z^{(n)}(t)\right]^{\alpha}\right| \leq r$ for all $\mathrm{n}$ and a.e. $t \in J$, so $z^{(n)} \in B_{q}$ and $z \in B_{q}$. By note we have

$$
\left\|z_{t}^{(n)}+y_{t}\right\| \leq q^{\prime}, \quad t \in J
$$

From conditions $\left(\mathrm{A}_{2}\right)-\left(\mathrm{A}_{6}\right)$, we have

(1) $I_{k}, k=1,2, \ldots, m$, is continuous.

(2) $h^{\alpha}\left(t, z_{t}^{(n)}+y_{t}\right) \rightarrow h^{\alpha}\left(t, z_{t}+y_{t}\right)$ for each $t \in J$ and since

$$
\left\|h^{\alpha}\left(t, z_{t}^{(n)}+y_{t}\right)-h^{\alpha}\left(t, z_{t}+y_{t}\right)\right\|<2\left[L_{1}^{\alpha}\|y\|+L_{2}^{\alpha}\right] .
$$

(3) $f^{\alpha}\left(s, z_{s}^{(n)}+y_{s}\right) \rightarrow f^{\alpha}\left(s, z_{s}+y_{s}\right)$ for each $s \in J$ and since $\left\|f^{\alpha}\left(s, z_{s}^{(n)}+y_{s}\right)-f^{\alpha}\left(s, z_{s}+y_{s}\right)\right\|<2 \lambda_{q}^{\alpha}(s)$.

From the dominated convergence theorem, we obtain

$$
\begin{aligned}
& \left|\left[(\Psi z)^{(n)}\right]^{\alpha}-[(\Psi z)]^{\alpha}\right|_{B_{h}} \\
& \quad \leq\left\|h^{\alpha}\left(t, z_{t}^{(n)}+y_{t}\right)-h^{\alpha}\left(t, z_{t}+y_{t}\right)\right\|+M_{1}^{\alpha} \int_{0}^{t} \| A(s) h^{\alpha}\left(s, z_{s}^{(n)}+y_{s}\right)
\end{aligned}
$$




$$
\begin{aligned}
& -A(s) h^{\alpha}\left(s, z_{s}+y_{s}\right) \| d s+\frac{1}{\beta}\left[M_{1}^{2}\right]^{\alpha}\left[M_{A}^{2}\right]^{\alpha} \int_{0}^{a}\left[\| h^{\alpha}\left(a, z_{a}^{(n)}+y_{a}\right)\right. \\
& -h^{\alpha}\left(a, z_{a}+y_{a}\right) d s\left\|+M_{1}^{\alpha} \int_{0}^{a}\right\| A(s) h^{\alpha}\left(a, z_{a}^{(n)}+y_{a}\right) \\
& -A(s) h^{\alpha}\left(a, z_{a}+y_{a}\right)\left\|d s+M_{1}^{\alpha} \int_{0}^{a}\right\| f^{\alpha}\left(s, z_{s}^{(n)}+y_{s}\right)-f^{\alpha}\left(s, z_{s}+y_{s}\right) \| d s \\
& +M_{1}^{\alpha} \int_{0}^{a}\left\|g^{\alpha}\left(s, z_{s}^{(n)}+y_{s}\right)-g^{\alpha}\left(s, z_{s}+y_{s}\right)\right\| d W(s) \\
& +M_{1}^{\alpha} \sum_{k=1}^{m} \| I_{k}^{\alpha}\left(z^{(n)}\left(t_{k}^{-}\right)+y\left(t_{k}^{-}\right)-I_{k}^{\alpha}\left(z\left(t_{k}^{-}\right)+y\left(t_{k}^{-}\right)\right) \|\right] d \eta \\
& +M_{1}^{\alpha} \int_{0}^{t}\left\|f^{\alpha}\left(s, z_{s}^{(n)}+y_{s}\right)-f^{\alpha}\left(s, z_{s}+y_{s}\right)\right\| d s \\
& +M_{1}^{\alpha} \int_{0}^{t}\left\|g^{\alpha}\left(s, z_{s}^{(n)}+y_{s}\right)-g^{\alpha}\left(s, z_{s}+y_{s}\right)\right\| d W(s) \\
& +M_{1}^{\alpha} \sum_{o<t_{k}<t} \| I_{k}^{\alpha}\left(z^{(n)}\left(t_{k}^{-}\right)+y\left(t_{k}^{-}\right)-I_{k}^{\alpha}\left(z\left(t_{k}^{-}\right)+y\left(t_{k}^{-}\right)\right) \| \rightarrow 0 \quad \text { as } n \rightarrow \infty\right.
\end{aligned}
$$

which shows that $\Psi$ is continuous in $B_{h}^{\prime \prime}$. Hence the conditions of Schauder's fixed point theorem are all satisfied, and also the operator $\Psi$ has a fixed point in $B_{q}$. Thus system (1)-(3) has a solution on $J$.

Theorem 3.2 Conditions $\left(\mathrm{A}_{1}\right),\left(\mathrm{A}_{3}\right),\left(\mathrm{A}_{5}\right)$ and $(\mathrm{D})$ are satisfied. Then system (1)-(3) is approximately controllable on $\mathrm{J}$.

Proof Let $\tilde{x}^{\gamma}(\cdot)$ be a fixed point of $\Psi$ in $B_{q}$, and any fixed point of $\Psi$ is a mild solution of (1)-(3) on $[0, b]$.

By Theorem 2.7, the control

$$
\tilde{u}^{\gamma}(t)=B^{*} U^{*}(a, t) \tilde{R}\left(\gamma, \Gamma_{0}^{a}\right)\left[P\left(\tilde{x}^{\gamma}\right)\right]^{\alpha},
$$

which satisfies

$$
\begin{aligned}
{\left[\tilde{x}^{\gamma}(a)\right]^{\alpha}=} & x_{a}-\gamma \tilde{R}\left(\gamma, \Gamma_{0}^{a}\right)\left[x_{a}-U^{\alpha}(a, 0)\left[x_{0}-\mu(x)-h^{\alpha}\left(0,\left(x_{0}-\mu(x)\right)\right)\right]\right. \\
& -h^{\alpha}\left(a, x_{a}\right)-\int_{0}^{a} U^{\alpha}(a, s) A(s) h^{\alpha}\left(s, \tilde{x}_{s}^{\gamma}\right) d s-\int_{0}^{a} U^{\alpha}(a, s) f^{\alpha}\left(s, \tilde{x}_{s}^{\gamma}\right) d s \\
& \left.-\int_{0}^{a} U^{\alpha}(a, s) g^{\alpha}\left(s, \tilde{x}_{s}^{\gamma}\right) d W(S)-\sum_{k=1}^{m} U^{\alpha}\left(a, t_{k}\right)\left(I_{k}\right)\left(x\left(t_{k}^{-}\right)\right)\right] .
\end{aligned}
$$

By $\left(\mathrm{A}_{3}\right)-\left(\mathrm{A}_{5}\right)$

$$
\begin{aligned}
& \int_{0}^{a}\left\|f^{\alpha}\left(s, \tilde{x}_{s}^{\gamma}\right)\right\| d s \leq L_{f}^{\alpha} a, \quad \int_{0}^{a}\left\|g^{\alpha}\left(s, \tilde{x}_{s}^{\gamma}\right)\right\| d W(s) \leq L_{g}^{\alpha} a, \\
& \int_{0}^{a}\left\|A(s) h^{\alpha}\left(s, \tilde{x}_{s}^{\gamma}\right)\right\| d s \leq L_{h}^{\alpha} a .
\end{aligned}
$$


Here, the sequences $f^{\alpha}\left(s, \tilde{x}_{s}^{\gamma}\right), g^{\alpha}\left(s, \tilde{x}_{s}^{\gamma}\right)$ and $A(s) h^{\alpha}\left(s, \tilde{x}_{s}^{\gamma}\right)$ are bounded in $L^{2}(J, X)$. Therefore, there is a subsequence $f^{\alpha}\left(s, \tilde{x}_{s}^{\gamma}\right), g^{\alpha}\left(s, \tilde{x}_{s}^{\gamma}\right)$ and $A(s) h^{\alpha}\left(s, \tilde{x}_{s}^{\gamma}\right)$ are all weakly convergent to say $f^{\alpha}(s), g^{\alpha}(s)$ and $A(s) h^{\alpha}(s)$ are bounded in $L^{2}(J, X)$, respectively. Take

$$
\begin{aligned}
\tilde{\omega}= & x_{a}-U^{\alpha}(a, 0)\left[x_{0}-\mu(x)-h^{\alpha}\left(0,\left(x_{0}-\mu(x)\right)\right)\right]-h^{\alpha}(a, x(a)) \\
& -\int_{0}^{a} U^{\alpha}(a, s) A(s) h^{\alpha}(s) d s \\
& -\int_{0}^{a} U^{\alpha}(a, s) f^{\alpha}(s) d s-\int_{0}^{a} U^{\alpha}(a, s) g \alpha(s) d W(S)-\sum_{k=1}^{m} U^{\alpha}\left(a, t_{k}\right) I_{k}^{\alpha}\left(x\left(t_{k}^{-}\right)\right) .
\end{aligned}
$$

It follows by the compactness of the operators $q(\cdot) \rightarrow \int_{0}^{a} U(\cdot, s) q(s) d s: L^{2}(J, X) \rightarrow C(J, X)$, we obtain that

$$
\begin{aligned}
\left\|\left[P\left(\tilde{x}^{\gamma}\right)\right]^{\alpha}-\tilde{\omega}\right\| \leq & \left\|\int_{0}^{a} U^{\alpha}(a, s)\left[A(s) h^{\alpha}\left(s, \tilde{x}_{s}^{\gamma}\right)-A(s) h^{\alpha}(s)\right] d s\right\| \\
& +\left\|\int_{0}^{a} U^{\alpha}(a, s)\left[f^{\alpha}\left(s, \tilde{x}_{s}^{\gamma}\right)-f^{\alpha}(s)\right] d s\right\| \\
& +\left\|\int_{0}^{a} U^{\alpha}(a, s)\left[g^{\alpha}\left(s, \tilde{x}_{s}^{\gamma}\right)-g^{\alpha}(s)\right] d W(S)\right\| \rightarrow 0 \quad \text { as } \gamma \rightarrow 0^{+} .
\end{aligned}
$$

By (11) and definition

$$
\begin{aligned}
\left\|\left[\tilde{x}^{\gamma}(a)\right]^{\alpha}-x_{a}\right\| & =\left\|\gamma \tilde{R}\left(\gamma, \Gamma_{0}^{a}\right)\left[P\left(\tilde{x}^{\gamma}\right)\right]^{\alpha}\right\| \\
& =\left\|\gamma \tilde{R}\left(\gamma, \Gamma_{0}^{a}\right)\left[P\left(\tilde{x}^{\gamma}\right)\right]^{\alpha}-\tilde{\omega}+\tilde{\omega}\right\| \\
& \leq\left\|\gamma \tilde{R}\left(\gamma, \Gamma_{0}^{a}\right) \tilde{\omega}\right\|+\left\|\gamma \tilde{R}\left(\gamma, \Gamma_{0}^{a}\right)\left[P\left(\tilde{x}^{\gamma}\right)\right]^{\alpha}-\tilde{\omega}\right\| \\
& \leq\left\|\gamma \tilde{R}\left(\gamma, \Gamma_{0}^{a}\right) \tilde{\omega}\right\|+\left\|\left[P\left(\tilde{x}^{\gamma}\right)\right]^{\alpha}-\tilde{\omega}\right\| \rightarrow 0 \quad \text { as } \gamma \rightarrow 0^{+},
\end{aligned}
$$

i.e., $\left\|\left[\tilde{x}^{\gamma}(a)\right]^{\alpha}-x_{a}\right\| \rightarrow 0$.

This proves the approximate controllability of (1)-(3).

\section{Example}

We consider an impulsive neutral fuzzy stochastic differential equation with nonlocal condition:

$$
\begin{aligned}
&{ }^{c} D_{t}^{\alpha} {\left[z(t, x)-\int_{-\infty}^{t} e^{(s-t)} z(s, x) d s\right] } \\
&= \frac{\partial^{2}}{\partial z^{2}}\left[z(t, x)-\int_{-\infty}^{t} e^{(s-t)} z(s, x) d s\right]+\eta(t, x) \\
& \quad \quad \int_{-\infty}^{t} \hat{a}(s) \sin z(t+s, x) d s+\int_{-\infty}^{t} \alpha(t-s) z(s, x) d W(s), \quad x \in[0, \Pi], \\
& z\left(t_{k}^{+}, x\right)-z\left(t_{k}^{-}, x\right)=I_{k}\left(z\left(\tau_{k}^{-}, x\right)\right), \quad k=1,2, \ldots, n, \\
& z(t, 0)=z(t, \Pi)=0, \quad t \in J=[0, b], \\
& z(0, x)+\int_{0}^{\Pi} k_{1}(x, y) z(t, y) d y=\phi(t, x), \quad t \in(-\infty, 0] .
\end{aligned}
$$


Let $U=L^{2}([0, \Pi])$ and $v(t)=e^{2 t}, t>0$, where ${ }^{c} D_{t}^{\alpha}$ is a Caputo fractional partial derivative of order $0<\alpha<1, b>0, k_{1}(x, y) \in L_{2}([0, \Pi] \times[0, \Pi])$ and $\int_{-\infty}^{0}|\hat{a}(s)| d s<\infty$. In our formulation we take $U$ defined on a fuzzy stochastic space $(\Omega, F, P)$. To study the approximate controllability of (12)-(15), assume that $\alpha(t)$ is measurable and continuous with finite $L_{\alpha}^{2}=\int_{-\infty}^{0} \frac{\alpha^{2}(-s)}{v(s)} d s$. We define the operator $A$ by $A z=\frac{\partial^{2} z}{\partial x^{2}}$ with domain

$$
\begin{aligned}
D(A)= & \left\{z \in U \text { and } \frac{\partial z}{\partial x}, \frac{\partial^{2} z}{\partial x^{2}} \text { are absolutely continuous, } \frac{\partial^{2} z}{\partial x^{2}} \in U\right. \text { and } \\
& z(0)=z(\Pi)=0\} .
\end{aligned}
$$

From this we know that $A$ generates a strongly continuous semigroup $T(t), t>0$, given by $T(t) z=\sum_{n=1}^{\infty} e^{-n^{2} t}\left\langle z, e_{n}\right\rangle e_{n}$, which is compact, analytic, and also spectrum of $A$ consists of the eigenvalues $-n^{2}$ for $n \in N$ with corresponding eigenvectors $z_{n}(x)=\sqrt{\frac{2}{\Pi}} \sin (n x)$, and $z_{n}$ is an orthonormal basis of $H$.

Define the bounded linear operator $B$ by

$$
B u(t)(x)=\eta(t, x), \quad 0 \leq x \leq \Pi, u \in U .
$$

Now, we present a special space $C_{v}$. For $(t, \varphi) \in J \times C_{v}$, where $z(s, x)=\varphi(s, x) \in(-\infty, 0] \times$ $[0, \Pi]$, and the operator $h, f: J \times C_{v} \rightarrow U, g: J \times C_{v} \rightarrow L_{2}^{0}(U, U)$ by

$$
\begin{aligned}
& h(t, \varphi)(x)=\int_{-\infty}^{0} e^{-s} \varphi(s, x) d s, \quad f(t, \varphi)(x)=\int_{-\infty}^{0} \hat{a}(s) \sin (\varphi(s, x)) d s, \\
& g(t, \varphi)(x)=\int_{-\infty}^{0} \alpha(-s) \varphi(s, x) d W(s)
\end{aligned}
$$

with the choice of $A, h, f, g$ of system (12)-(15) can be rewritten as the abstract form of system (1)-(3). Thus, under the appropriate conditions on the function $h, f, g$ and $I_{k}$ as those in $\left(A_{1}\right)-\left(A_{6}\right)$, system (12)-(15) is approximately controllable.

\section{Conclusion}

In this paper, we focused on approximate controllability results for impulsive neutral fuzzy stochastic differential equations with nonlocal conditions in a Banach space by using Schauder's and Banach fixed point theorems. According to the hypotheses, it is proved that the system is approximately controllable under nonlocal conditions. Then, the result showed that these fixed point theorems can strongly be used in control problems to obtain the sufficient conditions. Upon making some approximate assumptions, by the ideas and techniques as suggested in this paper, one can establish the approximate controllability results for a wide class of linear fuzzy stochastic evolution equations.

Competing interests

The authors declare that they have no competing interests.

\section{Authors' contributions}

All authors contributed equally to the writing of this paper. All authors read and approved the final manuscript. 


\section{References}

1. Negoita, CV, Ralescu, DA: Applications of Fuzzy Sets to System Analysis. Wiley, New York (1975)

2. Kwun, YC, Park, DG: Optimal control problem for fuzzy differential equations. In: Proceedings of the Korea-Vietnam Joint Seminar, pp. 103-114 (1998)

3. Mao, XR: Stochastic Differential Equations and Their Application (1997)

4. Ruan, S: Oscillation of second order neutral differential equations. Can. Math. Bull. 36, 485-496 (1991)

5. Zhang, X, Feng, M: Transformation technique, fixed point theorem and positive solutions for second-order impulsive differential equations with deviating arguments. Adv. Differ. Equ. 2014, 312 (2014)

6. Zhang, MR: Periodic solutions of linear and quasilinear neutral functional differential equations. J. Math. Anal. Appl. 189(2), 378-392 (1995)

7. Benchohra, M, Henderson, J, Ntouyas, S: Impulsive Differential Equations and Inclusions. Contemporary Mathematics and Its Applications, vol. 2 (2006)

8. Lakshmikantham, V, Bainov, DD, Simeonov, PS: Theory of Impulsive Differential Equations. World Scientific, Singapore (1989)

9. Dauer, JP, Mahmudov, NI, Matar, MM: Approximate controllability of backward stochastic evolution equations in Hilbert spaces. J. Math. Anal. Appl. 323, 42-56 (2006)

10. Dauer, JP, Mahmudov, NI: Approximate controllability of semilinear functional equations in Hilbert spaces. J. Math. Anal. Appl. 273, 310-327 (2002)

11. Sakthivel, R, Mahmudov, NI, Kim, JH: Approximate controllability of nonlinear impulsive differential systems. Rep. Math. Phys. 60, 85-96 (2007)

12. Sakthivel, R, Nieto, J, Mahmudov, NI: Approximate controllability of nonlinear deterministic and stochastic systems with unbounded delay. Taiwan. J. Math. 14(5), 1777-1797 (2010)

13. Zang, Y, Li, J: Approximate controllability of fractional impulsive neutral stochastic differential equations with nonlocal conditions. Bound. Value Probl. 2013, 193 (2013)

\section{Submit your manuscript to a SpringerOpen ${ }^{\odot}$ journal and benefit from:}

- Convenient online submission

- Rigorous peer review

- Immediate publication on acceptance

- Open access: articles freely available online

- High visibility within the field

- Retaining the copyright to your article 